\title{
Remembering and the prefrontal cortex
}

\author{
RICHARD S. LEWIS \\ Pomona College, Claremont, California
}

\begin{abstract}
This paper comments on a recent article by Daniel L. Schacter (1987) that reviewed the function of the frontal lobes in remembering. Schacter concluded that the frontal lobes are involved in spatiotemporal contextual processing of memorial information, and that this aspect of context is processed in a relatively automatic fashion. The present paper supports Schacter's contention that the frontal lobes are involved in the temporal context of memory, but their role in the spatial context is questioned, given its limited empirical support. It is, instead, suggested that structures in the medial temporal lobe region, primarily the hippocampus, may play a more critical role in processing spatial contextual memory.
\end{abstract}

Speculations regarding the involvement of the frontal lobes in memory extend at least back to Gall (1835), who claimed that verbal memory and the memory for persons were localized in the area above and behind the eyes. In Gall's view, though, memory was not considered to be a fundamental function; rather, it was thought to be a secondary property of fundamental functions such as calculating, locality, and music. He, therefore, endorsed a multiple memory system model, and believed that any function located in the frontal lobes (this terminology and designation of the cortical lobes was not in use at that time) would also be involved in the remembrance associated with that function. Following Gall, memory impairment after frontal lobe damage was observed in clinical studies in the early and middle part of this century (Kolodny, 1929; Rylander, 1939; Stookey, Scarff, \& Teitelbaum, 1941). Interestingly, though, no problems with memory were specifically noted in the case of Phineas Gage, the celebrated study of frontal lobe damage that resulted from a tamping iron accident (Macmillian, 1986). Although memory is an important consideration in models of frontal lobe functioning (e.g., Fuster, 1980; Milner, Petrides, \& Smith, 1985; Stuss \& Benson, 1986), and the idea of a relationship between the frontal lobes and memory has been around for well over a century, relatively little attention has been paid to frontal lobe involvement in formal models of memory.

Recently, Schacter (1987) has argued that studying the role of the frontal lobes in memory will be useful in gaining a more complete understanding of how the brain processes memories. Schacter proposes that the frontal lobe is involved in the memory of spatiotemporal infor-

The author would like to extend his appreciation to Deborah Burke and Kathi Hirsh for the many valuable comments offered on an earlier draft of this manuscript. The author's mailing address is Department of Psychology, Pomona College, Claremont, CA 91711-6358. mation, and that this information is processed relatively automatically. In his review, Schacter analyzes three types of research: (1) the impact of frontal lobe pathology on amnesic symptomatology, (2) memory dysfunction in patients with frontal lobe pathology, and (3) cognitive deficits in animals with frontal lobe lesions. In the final section of Schacter's article, he considers whether spatiotemporal information is processed automatically.

I fully concur with Schacter's (1987) claim that the literature supports the role of the frontal lobes in the processing of temporal contextual information. However, I question his contention that the frontal lobes also play a role in the spatial context of memories.

The present comment will follow the format of Schacter's (1987) review and will address his primary points in the order presented in his article. The first question to be considered, therefore, is to what extent the literature supports the role of a spatiotemporal memory deficit in amnesics with frontal lobe pathology. For the most part, Schacter accomplishes this task by comparing amnesics with purported frontal lobe dysfunction (e.g., Korsakoff patients) with amnesics who showed no evidence of frontal lobe impairment (e.g., temporal lobectomy patients).

Korsakoff amnesics have been found to perform poorly on tests of frontal lobe functioning (Moscovitch, 1982; Squire, 1982). Consequently, as Schacter (1987) points out, Korsakoff patients (as well as other amnesics with evidence of frontal lobe dysfunction) may manifest unique cognitive impairment compared with amnesics who show no evidence of frontal lobe dysfunction. The literature cited by Schacter strongly supports the involvement of the frontal lobes in the temporal aspects of memory but, as will be shown, support for the involvement of the frontal lobes in the spatial aspects of memory functions is relatively weak.

Studies have demonstrated that Korsakoff patients are impaired in temporal discrimination of verbal material 
(Squire, 1982), temporal discrimination of pictures (Huppert \& Piercy, 1976), recency judgments (Meudell, Mayes, Ostergaard, \& Pickering, 1985), and proactive interference (Kinsbourne \& Winocur, 1980; Winocur \& Weiskrantz, 1976). An additional study cited by Schacter and his colleagues (Schacter, Harbluk, \& McLachlan, 1984) reported that amnesics with mixed etiologies (dementia of the Alzheimers type, anoxia, closed head injury, encephalitis, ruptured anterior communicating artery, and one undiagnosed) showed deficits in making judgments about the source of fictitious information. However, such a task confounds temporal and spatial information, and it is therefore difficult to determine if source amnesia is due to a selective deficit of temporal or spatial contextual information (or other aspects of context), a deficit in the combination of temporal and spatial information, or some other factor.

If the temporal discrimination deficit is specific to amnesics with frontal lobe dysfunction, then it should not be present in amnesics in whom there is no suspicion of frontal lobe impairment. This has been shown to be the case with N.A., a patient with a verbal memory deficit predominantly due to a diencephalic lesion, and with patients undergoing ECT therapy for depression, for judging on which of two days sentences were presented (Squire, 1982). The specificity of a temporal discrimination deficit for amnesics with suspected frontal lobe involvement is difficult to evaluate in other studies because of the confounding factor of etiology often present in those studies. For example, Warrington and Weiskrantz (1974, 1978) found that their group of amnesics showed increased proactive interference, but only 2 out of the 4 amnesics in that study were of the Korsakoff variety; the other 2 were suspected to have temporal lobe involvement. Similarly, Hirst and Volpe (1982) found that a group of amnesics with mixed etiologies (hypoxia, anterior communicating artery rupture, stroke, and closed head injury) were impaired in judging the temporal order of words and news events. Interestingly, though, the patients in that study did not show impairment on traditional frontal lobe measures, such as the Wisconsin Card Sorting Test and verbal fluency.

The evidence cited by Schacter (1987) supports the hypothesis that amnesics with suspected frontal lobe dysfunction show deficits in the temporal aspects of contextual memory. The specificity of this dysfunction is less clear, though, largely because of the varied etiologies of amnesia in some of the studies. However, where the evidence is available, for example in Squire's (1982) study cited above, it is consistent with the hypothesis that temporal discrimination deficits are not found in amnesics who show no evidence of frontal lobe dysfunction.

It should be noted at this point that the amnesics who showed deficits of temporal contextual memory also tended to have lesions of the mediodorsal thalamus (MD). Considering the close anatomical and functional connections between MD and the prefrontal cortex, (the prefrontal cortex, as well as its subdivisions, can be defined by the MD projections), it is important to consider whether the temporal contextual deficit is part of a larger dysfunction of the MD-prefrontal system. Data from patients with selective damage to the MD are inconclusive. Spiegel, Wycis, Orchinik, and Freed (1955) reported the presence of a transient temporal confusion in a series of patients who underwent MD thalamotomies for the relief of "emotional disturbances" or pain. In contrast, N.A., the patient with reported damage to the left MD, performed significantly better than chance on a measure of temporal order (Squire, 1982). This result must be cautiously interpreted, however, because: (1) the result is from a single subject, (2) the subject was not globally amnesic (by virture of his lateralized lesion, his memory deficit was limited to verbal information), and (3) the site of his proposed MD lesion has been questioned by Weiskrantz (1985). In support of Weiskrantz, a recent magnetic resonance imaging scan revealed more extensive damage to N.A. than was originally thought based on the result of CT scans. Damage was reported to include the left diencephalon, bilateral mammillary bodies, and the right anterior temporal lobe (Squire, Amaral, Zola-Morgan, Kritchevesky, \& Press, 1988). There is, therefore, little conclusive evidence of the role of the MD in temporal memory; this relationship needs to be examined in future studies.

Since Schacter (1987) proposes that the frontal lobes are involved in the temporal and spatial aspects of contextual memory, it is important to consider the evidence supporting the frontal lobes' involvement in spatial contextual memory. Schacter cites an experiment by Winocur and Kinsbourne (1978) as demonstrating "that Korsakoff patients also have problems using features of spatial context to aid memory performance." In that study, Korsakoff amnesics and alcoholic controls were given two lists of paired associates. The stimulus words were the same on both lists, whereas the response words were different. In their first experiment, Winocur and Kinsbourne found there was no difference between the two groups of subjects in the number of errors for the first list of paired associates, but on the second list the amnesics made significantly more errors than did the control group. Sixty-six percent of the errors for the amnesics were intrusion errors from the first list, which suggests a temporal contextual problem. In Experiment 2, the procedure was the same as in Experiment 1, except that the first list of paired associates was presented in a distinct environment (with a red light shining over the paired associate cards and classical music playing in the background), whereas the second list was presented in a more conventional laboratory environment (i.e., no red light and no background music). Winocur and Kinsbourne found no difference in performance for the control group between the List 2 condition in the first and second experiment. The amnesics, however, made significantly fewer errors in the "context-shift" condition, including a significant decrease in the number of intrusion errors from List 1. 
Although Schacter interprets these results to suggest that "Korsakoff patients have special difficulties in responding to environmental cues, difficulties that can be overcome when the patients are placed in a distinctive and unusual environmental context," I would instead interpret these results to suggest that a large part of the amnesics' problem in Experiment 1 was due to their difficulty with temporal discrimination between the two lists. Presumably, in Experiment 1, the subjects were tested with both lists in the same environment, and the only difference in the presentation between the two lists was in the temporal dimension. In Experiment 2, however, the difficulty in temporal discrimination was reduced because the two lists were presented in a different environmental context. It appears, therefore, that the Korsakoff patients could use a distinction in environmental context to aid in their memory. I would conclude that the results of these experiments add further support to the hypothesis that Korsakoff patients (often with signs of frontal lobe dysfunction) show a temporal contextual memory deficit, but have the ability to use environmental context to aid their memory. As such, these results parallel the findings of increased proactive interference found in Korsakoff patients (Kinsbourne \& Winocur, 1980; Winocur \& Weiskrantz, 1976), and provide further support for a temporal deficit in these patients.

An analogous result to proactive interference with verbal information was reported for spatial information by Schacter, Moscovitch, Tulving, McLachlan, and Freedman (1986). They found that in two spatial location experiments (one involving the location of objects in a room and the other involving the location of objects in different drawers of a container), amnesics with signs of frontal lobe dysfunction performed as well as controls (with no errors) in finding the objects at their initial locations. However, on subsequent trials, when the objects were placed in a new location, amnesics were impaired relative to the controls, and tended to search the old location. The amnesics' performance in attempting to look for the objects in their initial location suggests they did not have difficulty with memory involving spatial context (although such an effect may have been masked by a ceiling effect). However, the perseverative response or "mnemonic precedence" reported in this study does suggest a problem with temporal discrimination. Diamond and Goldman-Rakic (1983) reported a similar phenomenon in monkeys after prefrontal lesions (but no deficit was found after hippocampal lesions; Diamond, Zola-Morgan, \& Squire, 1988). It should be noted, however, that Schacter et al. (1986) failed to find evidence for mnemonic precedence in nonamnesic patients with frontal lobe lesions.

The second type of research considered by Schacter (1987) was on spatiotemporal contextual memory failures by nonamnesic patients with frontal lobe lesions. Again, the literature on the involvement of the frontal lobes in spatiotemporal contextual memory generally supports the idea that the frontal lobes are involved in temporal con- textual information, but provides little support for their involvement in a spatial context.

Schacter (1987) refers to reviews by Milner et al. (1985), Squire (1987), and Stuss and Benson (1986), which cited data supporting deficits in temporal order judgments and temporal discriminations in patients with frontal lobe lesions. For example, patients with frontal lesions performed poorly on recency judgments of verbal and nonverbal material (Corsi, as cited by Milner, 1971, 1974), recall of the temporal order of pictures and words (Lewinsohn, Zieler, Libet, Eyeberg, \& Nielson, 1972), reproduction of the temporal order of arm and facial movements (Milner \& Kolb, 1985), and frequency estimation of presented stimuli (Smith \& Milner, 1983). In addition, Schacter cites several studies that found that "patients with frontal damage also perform poorly when memory tasks require various types of sequential organization (della Rocchetta, 1986; Petrides \& Milner, 1982), recollection of autobiographical episodes with specific temporal dates (Baddeley \& Wilson, 1986), or accurate temporal discriminations (Prisko, cited in Milner, 1964)" (p. 26), as well as on delayed alternation, studies on patients with frontal damage requiring the subject to remember the response on the previous trial (Chorover \& Cole, 1966), a variety of alternation tasks that require temporal discrimination (Pribram, Ahumada, Hartog, \& Roos, 1964), and excessive proactive interference on the BrownPeterson task, which requires temporal discrimination of the trials (Stuss et al., 1982).

Given the evidence cited above, I would concur with Schacter's (1987) conclusion that

the foregoing studies provide converging evidence for the
view, proposed by various investigators (e.g., Diamond \&
Goldman-Rakic, 1983; Fuster, 1980; Goldman-Rakic, 1984;
Milner, 1971; Milner et al., 1985; Stuss et al., 1982), that
patients with relatively restricted frontal damage are impaired
on memory tasks that require utilization of temporal infor-
mation, and perform relatively normally on memory tasks
that do not make temporal demands. (p. 27 )

In the one study addressing the role of the frontal cortex in spatial memory cited in this section of Schacter's (1987) review, Smith and Milner (1984) did not find frontal patients to be impaired on a measure of recall of spatial location. Schacter discounts this study by questioning the relevance of this task to contextual information of a more global nature. He is correct in pointing out the inadequacies of Smith and Milner's definitions of spatial context (see also Mayes, Meudell, \& Pickering, 1985). Indeed, if one were inclined to force a dichotomy of spatial context, which is most likely a continuum, then distinguishing between local and global spatial context might be one reasonable approach. In such a paradigm, spatial location of an array of stimuli presented on a CRT or on a table would be more typical of local spatial context, whereas the room, building, or city location would be more typical of global contextual information. Such a dichotomy would certainly bring into question the 
relevance of Smith and Milner's (1983) lack of finding a spatial location deficit of objects scattered on a table relative to the more global aspects of contextual information. However, if Schacter is to be consistent, then he might also distinguish between local and global aspects of temporal context; the former being more typical of the order of words presented in a list, for example, and the latter being represented by the day or year in which an event occurred. This approach would call into question, perhaps, the relevance of many of the research studies cited above to the more global aspects of temporal contextual memory. It remains to be determined what the relationship is between the different aspects of temporal and spatial context (i.e., global vs. local). In any event, in this section, Schacter does not present any evidence to support the role of frontal lobe lesions in impaired spatial memory.

The third section of Schacter's (1987) article concerns cognitive deficits in animals with frontal lobe lesions. Schacter outlines three types of procedures often used to measure memory functions in animals: (1) delayed response tasks, which involve recall of where bait is placed after a delay period; (2) delayed alternation tasks, which involve remembering the location of bait on the previous trial; and (3) delayed matching to sample tasks, which involve recalling the most recently presented stimulus. Monkeys with lesions to the frontal cortex have been shown to be impaired on all of these tasks. All three of these tasks have significant temporal components, and all except the delayed matching to sample task have significant spatial components. That is, delayed response and delayed alternation tasks confound the temporal and spatial components of memory. The results of the delayed matching to sample studies, therefore, suggest that a task with only a temporal contextual component is sufficient for a memory deficit in animals with frontal lesions. As Schacter states, an interesting question becomes, "Would animals with frontal damage perform poorly on memory tasks-spatial or otherwise-that do not have a significant temporal component?' ' (p. 28). In an attempt to answer this question, he considers studies that used trial-unique stimuli, in contrast to the majority of animal studies, which used the same stimuli repeatedly over many trials. Such an approach addresses the question of whether a memory deficit after frontal lobe lesions would be present on tests without a temporal component, but does not necessarily examine whether the frontal lobe is involved in spatial contextual information. Schacter cites Meyer, Harlow, and Settlage (1951) as finding that "monkeys with bilateral frontal lobe lesions performed better in the trialunique situation than in the standard task with recurring stimuli, but that the performance of monkeys with unilateral lesions or no lesions did not differ in the two tasks" (pp. 28-29). Furthermore, he cites Mishkin and Bachevalier (1983) as finding that monkeys with dorsolateral frontal lesions were not significantly impaired on the trial-unique procedure, but that monkeys with ventromedial lesions were impaired. Schacter, therefore, concludes that there is converging evidence from human and animal data that dorsolateral lesions "impair performance on tasks that require temporal memory but apparently do not do so on ones that require nontemporal memory" (p. 29).

From the three previous sections of his review, it appears that Schacter's (1987) sole source of support for frontal involvement in spatial contextual memory comes from animal studies that found that monkeys were more impaired on tasks that involved temporal and spatial components than on tasks that involved only a temporal component. It remains to be demonstrated, however, that the greater impairment was due specifically to the addition of a spatial component, rather than to that the combined processes that reflect greater task demands, difficulty, or complexity.

In the final section of the review, Schacter (1987) convincingly argues that spatiotemporal information is processed in a relatively automatic fashion, and he suggests that the contextual memory failure found in patients with frontal lobe lesions was not an indirect consequence of a generalized deficit in effortful processing. It is important to keep in mind, though, that the automaticity of spatial location and temporal order have been questioned with respect to the strict criteria proposed by Hasher and Zacks (1979) (see, e.g., Puglisi, Park, Smith, \& Hill, 1985; Zacks, Hasher, Alba, Sanft, \& Rose, 1984). Indeed, it may be that in a continuum, these processes are more typical of automatic than of effortful processes.

Despite Schacter's (1987) conclusion that the frontal lobes are involved in the spatiotemporal processing of mnemonic information, his review provides strong support only for the conclusion that the frontal lobes are involved in the memory of temporal contextual information. It is therefore important to avoid the tendency to group spatial and temporal contextual memory together. The term spatiotemporal context may be misleading in this respect. Certainly these two aspects of context are conceptually distinguishable, and there is evidence that they are also functionally distinguishable. For example, in a simple demonstration of the functional independence of spatial and temporal memory, one of my students, Lori Shaler, investigated, for her undergraduate thesis, the rates of forgetting for temporal order and spatial location. In that study, slides of objects, buildings, and landscapes were presented on one of three screens to the left, right, or center of the 17 experimental participants in an explicit memory paradigm. The subjects were tested at three delays (5 min, 4 days, and 1 week following the presentation of the stimuli) for their recognition of the slides (distractor slides were from the same category as the targets). If they recognized a slide as having been previously presented, the subjects were asked in which third of the list it had been presented and on which of the three screens it had appeared. In relation to the accuracy of the recall of location and time of stimulus presentation, a significant interaction was found between delay and the nature of the contextual condition $[F(2,34)=13.03$, $p<.0001]$, which suggests a functional independence between temporal and spatial contexts. 
The spatial and temporal contexts explored in this study represent the more local aspects of context described above, and therefore may not address the more global aspects of context (though, again, the relationship between the two has not been adequately addressed). It should be recognized that investigations of the more global aspects of context tend to confound spatial and temporal context. That is, any change in global spatial context (such as moving from one room to the next) necessarily involves a change in temporal context. This concept is clearly articulated in Fuster's (1980) monograph on the prefrontal cortex. He views the functions of the prefrontal lobe as being comprised as a hierarchy, and postulates that the superordinate function is the temporal structuring of behavior. For the processing of spatial information, Fuster believes that

such a role is subordinate to the temporal synthetic function.... There are two reasons for that belief: first, spatial discontiguity in perception is to a great extent reducible to temporal discontiguity; second, even lesions of that small cortical area (within the dorsolateral prefrontal cortex) disrupt only spatial tasks that include a delay within each trial. (p. 132)

This interpretation would lead us to expect deficits in spatial contextual memory after frontal lobe lesions, but only when a temporal component is also present.

With respect to global context, it is possible to isolate the temporal contextual component, as has been achieved in some of the studies cited above. It is, however, difficult to isolate the global aspects of spatial context without confounding the factor of temporal context. It is perhaps for this reason that many studies have chosen to investigate context in a more general sense, and have lumped the two terms together, or have tended to minimize the problem by investigating the more local aspects of temporal and spatial context as did Smith and Milner (1984).

If temporal and spatial context are conceptually and functionally separable components, then we might expect them to be represented by different (though perhaps overlapping) anatomical systems. An anatomical candidate for the processing of spatial context might be the medial temporal lobe area (see, e.g., Lewis, 1986). The medial temporal lobe region contains the hippocampus, which has been proposed to play a critical role in the spatial aspects of memory (e.g., O'Keefe \& Nadel, 1978). In support of this argument, patients with unilateral temporal lobectomies, as well as one with a bilateral temporal lobectomy, were reported to show a deficit for relocating objects on a table (Smith, 1988; Smith \& Milner, 1983). (Recall that Smith and Milner, 1984, found that patients with frontal lobe damage were not impaired on this task.) In addition, Parkinson and Mishkin (1982) found that monkeys with hippocampal lesions (but not monkeys with amygdalectomies) were impaired on a spatial location task. Furthermore, Holmes, Jacobson, Stein, and Butters (1982) reported that monkeys with mammillary body lesions (the mammillary bodies have close functional con- nections with the hippocampus) were impaired on a spatial memory task.

It should be noted that the hippocampus has reciprocal connections with the frontal lobe (Goldman-Rakic, Selemon, \& Schwartz, 1984). It is, therefore, possible that a specific region within the frontal lobe is responsible for processing the spatial context of memorial information. The primary point here is that this has yet to be adequately demonstrated with humans and nonhuman primates.

In the pursuit of understanding temporal and spatial context, we must keep in mind that space and time are just two aspects of context. A broader term that encompasses spatial context is environmental context, which refers to spatial context as well as other aspects of the stimulus environment, such as sights, smells, and sounds. In addition, one's internal state also appears to play a critical role in memory; take, for example, the phenomenon of state-dependent learning (e.g., Eich, 1980).

Although this commentary questions some of Schacter's (1987) conclusions, it is not meant to detract from perhaps the greatest contribution of his review-drawing more attention to understanding the role of the frontal lobes in memory. This commentary is, in fact, just a specific instance of a more general approach to the study of memory that is implied in Schacter's article, that is, studying how memory impairment is involved in different pathological conditions besides just amnesia. We have certainly profited to a great extent by studying amnesic subjects, but if we are to understand memorial processes and their relation to the brain fully, it will be necessary to take a more comparative view of memory disorders and attempt to investigate the role of memory functions in a variety of brain pathologies, as well as how memory interacts with other cognitive functions, such as language.

In summary, the present comment supports Schacter's (1987) conclusion that the prefrontal cortex (as well as perhaps the MD) is involved in the temporal contextual processing of memories, but questions its role in a spatial context. It is proposed, instead, that structures in the temporal lobe region (e.g., the hippocampus) may be more critically involved in spatial context. Complete agreement exists with Schacter's urging to broaden our perspective on memory by extending the scope of our attention to how other brain areas, outside of those most often implicated in the amnesic syndrome, are also involved in memory.

\section{REFERENCES}

Baddeley, A. D., \& Wilson, B. (1986). Amnesia, autobiograhical memory, and confabulation. In D. C. Rubin (Ed.), Autobiographical memory (pp. 225-252). New York: Cambridge University Press. Chorover, S. L., \& CoLE, M. (1966). Delayed alternation performance in patients with cerebral lesions. Neuropsychologia, 4, 1-7.

DELLA RoCCHetTA, A. I. (1986). Classification and recall of pictures after unilateral frontal or temporal lobectomy. Cortex, 22, 189-211. Diamond, A., Goldman-Rakic, P. (1983). Comparison of performance on a Piagetian object permanence task in human infants and rhesus monkeys: Evidence for involvement of prefrontal cortex. Society for Neuroscience Abstracts, 9, 641. 
Diamond, A., Zola-Morgan, S., \& SQuire, L. R. (1988). Successful performance by monkeys with hippocampal lesions on Piaget's AB task. Society for Neuroscience Abstracts, 13, 206.

EICH, J. E. (1980). The cue-dependent nature of state-dependent retrieval. Memory \& Cognition, 8, 157-173.

FUSTER, J. M. (1980). The prefrontal cortex: Anatomy, physiology and neuropsychology of the frontal lobe. New York: Raven.

GALL, F. J. (1835). On functions of the brain and each of its parts. (W. Lewis, Jr., Trans.). Boston: Margh, Capen \& Lyon.

Goldman-RaKIC, P. S. (1984). The frontal lobes: Unchartered provinces of the brain. Trends in Neurosciences, 7, 425-429.

Goldman-Rakic, P. S., Selemon, L. D., \& SchwartZ, M. L. (1984). Dual pathways connecting the dorsolateral prefrontal cortex with the hippocampal formation and parahippocampal cortex in the rhesus monkey. Neuroscience, 12, 719-743.

HAsHeR, L., ZACKS, R. T. (1979). Automatic and effortful processes in memory. Journal of Experimental Psychology: General, 108, 173-184.

HirsT, W., \& VolPE, B. T. (1982). Temporal order judgments with amnesia. Brain \& Cognition, 1, 294-306.

Holmes, E. J., Jacobson, S., Stein, B. M., Butters, N. (1982). Spatial memory and long-term retention following mammillary body lesions in monkeys. Society for Neuroscience Abstracts, 8, 23.

HupPert, F. A., \& PierCy, M. (1976). Recognition memory in amnesic patients: Effect of temporal context and familiarity of material. Cortex, 12, 3-20.

KinSBOURNE, M., \& WiNOcUR, G. (1980). Response competition and interference effects in paired-associate learning by Korsakoff amnesics. Neuropsychologia, 18, 541-548.

Kolodny, A. (1929). Symptomatology of tumor of the frontal lobe. Archives of Neurology \& Psychiatry, 21, 1107-1127.

Lewinsohn, P. M., Zieler, J. L., Libet, J., Eyeberg, S., \& Nielson, G. (1972). Short-term memory: A comparison between frontal and nonfrontal right- and left-hemisphere brain-damaged patients. Journal of Comparative \& Physiological Psychology, 81, 248-255.

LEWIS, R. S. (1986). Variation on a theme: Are the elements of episodic memory dissociable? Behavioral \& Brain Sciences, 9, 567-568.

Macmillian, M. B. (1986). A wonderful journey through skull and brains: The travels of Mr. Gage's tamping iron. Brain \& Cognition, 5, 67-107.

Mayes, A. R., Meudell, P. R., \& Pickering, A. (1985). Is organic amnesia caused by a selective deficit in remembering contextual information? Cortex, 21, 167-202.

Meudell, P. R., Mayes, A. R., Ostergaard, A., \& Pickering, A. (1985). Recency and frequency judgments in alcoholic amnesics and normal people with poor memory. Cortex, 21, 487-511.

Meyer, D. R., Harlow, H. F., Settlage, P. H. (1951). A survey of delayed performance by normal and brain-damaged monkeys. Journal of Comparative \& Physiological Psychology, 44, 17-25.

MilNER, B. (1964). Some effects of frontal lobectomy in man. In J. M. Warren \& K. Akert (Eds.), The frontal granular cortex and behavior (pp. 313-334). New York: McGraw-Hill.

MiLNER, B. (1971). Interhemispheric differences in the localization of psychological processes in man. British Medical Bulletin, 27, 272-277.

MilneR, B. (1974). Hemispheric specialization: Scope and limits. In F. O. Schmitt \& F. G. Worden (Eds.), The neurosciences: Third study program (pp. 75-89). Cambridge: MIT Press.

Milner, B., \& KolB, B. (1985). Performance of complex arm movements and facial-movement sequences after cerebral commissurotomy. Neuropsychologia, 23, 791-799.

Milner, B., Petrides, M., \& SMith, M. L. (1985). Frontal lobes and the temporal organization of memory. Human Neurobiology, 4, 137-142.

Mishkin, M., \& Bachevalier, J. (1983). Object recognition impaired by ventromedial but not dorsolateral prefrontal cortical lesions in monkeys. Society for Neuroscience Abstracts, 9, 29.

Moscovitch, M. (1982). Multiple dissociations of function in amnesia. In L. S. Cermak (Ed.), Human memory and amnesia (pp. 337370). Hillsdale, NJ: Erlbaum.

O'KEEFE, J., \& NADEL, L. (1978). The hippocampus as a cognitive map. New York: Oxford.
Parkinson, J. K., \& Mishkin, M. (1982). A selective mnemonic role for the hippocampus in monkeys: Memory for the location of objects. Society for Neuroscience Abstracts, 8, 23.

Petrides, M., \& Milner, B. (1982). Deficits on subject-ordered tasks after frontal and temporal lobe lesions in man. Neuropsychologia, 20, 249-262.

Pribram, K. H., Ahumada, A., Hartog, J., \& Roos, I. (1964). A progress report on the neurological processes disturbed by frontal lesions in primates. In J. M. Warren \& K. Akert (Eds.), The frontal granular cortex and behavior (pp. 28-55). New York: McGraw-Hill.

Puglisi, J. T., PARK, D. C., Smith, A. D., Hill, G. W. (1985). Memory for two types of spatial location: Effects of instructions, age, and format. American Journal of Psychology, 98, 101-118.

RyLANDER, G. (1939). Personality changes after operations on the frontal lobes: A clinical study of 32 cases. Acta Psychiatric Neurology, 20(Suppl.), 3-327.

SCHACTER, D. L. (1987). Memory, amnesia, and frontal lobe dysfunction. Psychobiology, 15, 21-36.

Schacter, D. L., Harbluk, J. L., \& Mclachlan, D. R. (1984). Retrieval without recollection: An experimental analysis of source amnesia. Journal of Verbal Learning \& Verbal Behavior, 23, 593-61 1.

Schacter, D. L., Moscovitch, M., Tulving, E., Mclachlan, D. R., FreEdMAN, M. (1986). Mnemonic precedence in amnesic patients: An analogue of the AB error in infants? Child Development, 57, 816-823.

Smith, M. L. (1988). Recall of spatial location by the amnesic patient H.M. Brain \& Cognition, 7, 178-183.

Smith, M. L., \& MiLner, B. (1983). Effects of focal brain lesions on sensitivity to frequency of occurrence. Society for Neuroscience $A b$ stracts, 9, 30.

Smith, M. L., \& Milner, B. (1984). Differential effects of frontal lobe lesions on cognitive estimation and spatial memory. Neuropsychologia, 22, 697-705.

Spiegel, E. A., Wycis, H. T., Orchinik, C. W., \&reed, H. (1955). The thalamus and temporal orientation. Science, 121, 771-772.

SQUIRE, L. R. (1982). Comparisons between forms of amnesia: Some deficits are unique to Korsakoff's syndrome. Journal of Experimental Psychology: Learning, Memory, \& Cognition, 8, 560-571.

Squire, L. R. (1987). Memory and brain. New York: Oxford.

Squire, L. R., Amaral, D. G., Zola-Morgan, S., KRItchEVesky, M., \& Press, G. (1988). New evidence of brain injury in the amnesic patient N.A. based on magnetic resonance imaging. Society for Neuroscience Abstracts, 13, 1454.

Stookey, B., SCARF, J., \& Teitelbaum, M. (1941). Frontal lobectomy in the treatment of brain tumors. Annals of Surgery, 113, 161-169.

Stuss, D. T., \& Benson, D. F. (1986). The frontal lobes. New York: Raven.

Stuss, D. T., Kaplan, E. F., Benson, D. F., Weir, W. S., Chiulli, S., \& Sarazin, F. F. (1982). Evidence of the involvement of orbitofrontal cortex in memory functions: An interference effect. Journal of Comparative \& Physiological Psychology, 96, 913-925.

Warrington, E. K., \& Weiskrantz, L. (1974). The effect of prior learning on subsequent retention in amnesic patients. Neuropsychologia, 12, 419-428.

WARRINGTON, E. K., \& WeISKRanTz, L. (1978). Further analysis of the prior learning effect in amnesic patients. Neuropsychologia, 16, 169-176.

WEISKRANTZ, L. (1985). On issues and theories of the human amnesic syndrome. In N. M. Weinberger, J. L. McGaugh, \& G. Lynch (Eds.), Memory systems of the brain. New York: Guilford.

Winocur, G. , \& KINSBourne, M. (1978). Contextual cueing as an aid to Korsakoff amnesics. Neuropsychologia, 16, 671-682.

Winocur, G., \&EISKRanTZ, L. (1976). An investigation of pairedassociate learning in amnesic patients. Neuropsychologia, 14, 97-110.

Zacks, R. T., Hasher, L., Alba, J. W., Sanft, H., \& Rose, K. C. (1984). Is temporal order encoded automatically? Memory \& Cognition, 12, 387-394.

(Manuscript received July 22, 1988; accepted for publication November 1, 1988.) 